\title{
Experimental study of EIT-Like phenomenon in a metamaterial plasma waveguide
}

\author{
Wusong Wang ${ }^{1}$, Liwei Zhang ${ }^{1,2}$, Kai Fang ${ }^{1}$, Yewen Zhang ${ }^{1 *}$ \\ ${ }^{1}$ MOE Key Laboratory of Special Artificial Microstructure Materials and Technology \\ Pohl Institute of Solid State Physics, Tongji University, Shanghai 200092, China \\ ${ }^{2}$ School of Physics and Chemistry, Henan Polytechnic University, Jiaozuo 454000, China \\ *corresponding author: yewen.zhang@tongji.edu.cn
}

\begin{abstract}
This paper demonstrates the realization of the electromagnetically induced transparency (EIT)-like transmission in a metamaterial plasma waveguide with double side defects based on transmission lines. The waveguide with a single side defect works as a plasma resonator and the resonance wavelength is determined by the Fabry-Perot resonance of surface plasma. But in a waveguide with double side defects, the destructive interference produces between the resonance modes of the two resonators. So a transmission peak appears between the two resonators frequencies, as indicates a pronounced EIT phenomenon. The experiment results agree well with simulations in microwave frequencies.
\end{abstract}

\section{Introduction}

The quantum phenomenon of electromagnetically induced transparency (EIT) has become a subject of intensive investigations in recent years [1]. It means regulating and controlling the optical response of the atomic medium by a secondary bunch of laser, which results in the emergence of a narrow transparent window within the original absorption spectrum. At the same time the abnormal dispersion in the transparent window caused by the two energy level resonation changes to the extremely intense normal dispersion [2, 3]. The EIT-related characteristics of strong dispersion and slow propagation within the transmitted frequency domains are showing many potential applications, such as nonlinear mixing and optical storage. But the original EIT was based on the quantum interference of atomic resonances, which makes it difficult to realize in experiments [4]. Recently, the EIT-like transmission based on metamaterials has been realized under simple experimental conditions $[5,6]$.

Metamaterials, including double negative materials (DNG, negative $\varepsilon$ and $\mu$ ) and single negative materials (one negative and one positive for $\varepsilon$ and $\mu$ ), have attracted intensive studies in the past few years [7, 8]. A medium with permittivity less than zero and permeability greater than zero is called $\varepsilon$-negative $(\mathrm{ENG})$ material. And a medium with the permittivity greater than zero and permeability less than zero is called $\mu$-negative (MNG) material. Metamaterials can be realized through L-C loaded transmission line structures [7, 8]. One of the most interesting properties is that the metamaterial structures support surface plasma, which is a kind of electromagnetic (EM) waves that propagate along the interface and decay in the transverse direction exponentially into both media. In general surface plasmon polaritons (SPPs) exist at optical frequencies. But they can also exist at microwave frequencies and they are named surface electromagnetic waves at this moment. That is to say surface electromagnetic waves are a mode of SPPs in microwave frequencies. In reference [9] Ruppin pointed out that SPPs appeared when the mathematical signs of permittivity and permeability are different which locate at the two sides of interface.

\section{Results of experiment and discussion}

In this paper, we demonstrate that EIT-like transmission based on the metamaterial plasma waveguide which is realized by using lumped element loaded transmission lines. As is shown in figure 1(a), a two dimensional metamaterials plasma waveguide with a single side defects with the sandwich structure (MNG-DPS-MNG) is fabricated. The MNG medium and the double positive ones are designed on a dielectric substrate (relative permittivity of 4.75) of height $\mathrm{h}=1.6 \mathrm{~mm}$ with a square grid (where the microstrip line width $\mathrm{w}=1 \mathrm{~mm}$ ) and the period is $\mathrm{d}=10 \mathrm{~mm}$. Unit series capacitance of $0.5 \mathrm{pF}$ is loaded in the $\mathrm{MNG}$ region and the double positive material (DPS) region is consisted of a row of microstrip lines in the middle of the waveguide. In order to prevent the reflection of EM waves by the boundary, the matched resistances of $85 \mathrm{ohm}$ are bridged between microstrip lines and ground level. According to the discussion in reference [9] the sandwich structure of MNGDPS-MNG support the emergence of SPPs. In the left MNG region (figure 1(a)), the region without shunt capacitance is the defect with length $\mathrm{L}_{1}=20 \mathrm{~mm}$ which is calibrated by an elliptically black curve. The input port and output one of electromagnetic (EM) waves are respectively connected through a section of microstrip line with length $\mathrm{L}=5 \mathrm{~mm}$ and width $\mathrm{w}^{\prime}=2.9 \mathrm{~mm}$. The transmission spectrum and electric field distribution of the waveguide with defect 
are simulated by Computer Simulation Technology (CST) and measured by means of an Agilent 8722ES vector network analyzer.

When only a single defect exists, the plasma waveguide is not transparent to the EM waves because the defect acts as a resonator. The plasma waveguide with the defect existing in the left MNG region is shown in figure 1 (a). Figure 1(b) shows the transmission spectrum and electric field distribution of the defective MNG-DPS-MNG plasma waveguide with defect length $\mathrm{L}_{1}=20 \mathrm{~mm}$ at the left side. The transparency $(\mathrm{dB})$ of simulation and measurement is in general accord and we obtained the transparency of $-30 \mathrm{~dB}$ at the frequency of $0.6 \mathrm{GHz}$ in measurement. The transparency of $-30 \mathrm{~dB}$ reveals that EM waves can hardly pass the palsma waveguide (Tranparency means the energy ratio of EM waves penetrating our waveduide). And the inset in figure 1(b) shows that our experimental structure is impenetrable to EM waves at the frequency of $0.6 \mathrm{GHz}$ because of the left defect acting as a Fabry-Perot resonance of surface plasma. The colors of red (or yellow) and blue represent respectively the positive and negative of the amplitude of the electric field intensity.

(a)

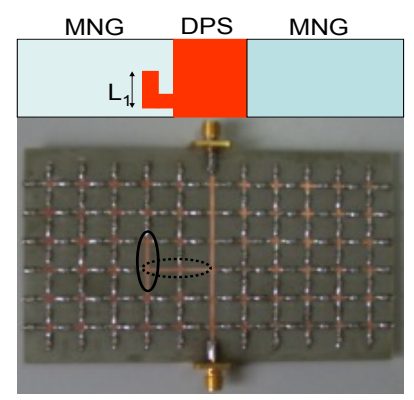

(b)

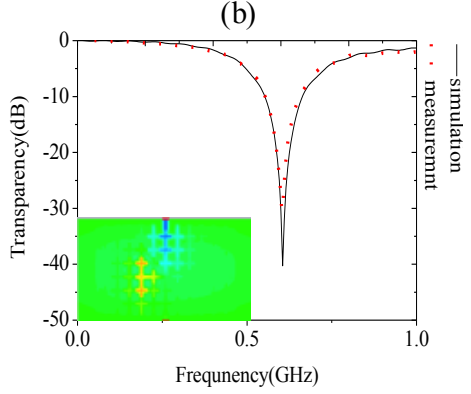

Figure 1:The plasma waveguide with defect length $\mathrm{L}_{1}=20 \mathrm{~mm}$ at the left side (a) and its transmission spectrum (b). The inset shows its electric field distribution.

We can also put the defect with length $\mathrm{L}_{2}=30 \mathrm{~mm}$ in the right $\mathrm{MNG}$ region, which is shown in figure 2(a). The transparency $(\mathrm{dB})$ of simulation and measurement is in general accord and we obtained the transparency of about $30 \mathrm{~dB}$ at the frequency of $0.5 \mathrm{GHz}$ (figure 2(b)) in measurement. And the result of CST simulation in the inset states clearly that our plasma waveguide is also impenetrable to EM waves at the frequency of $0.5 \mathrm{GHz}$. Here the resonant frequency is lower because the Fabry-Perot resonator become longer.

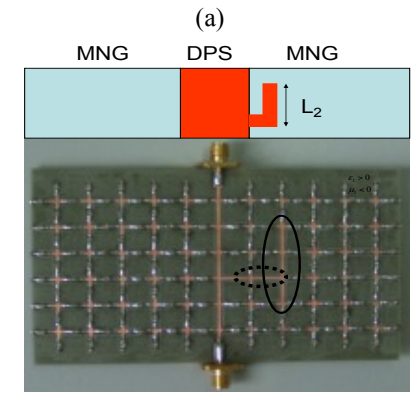

(b)

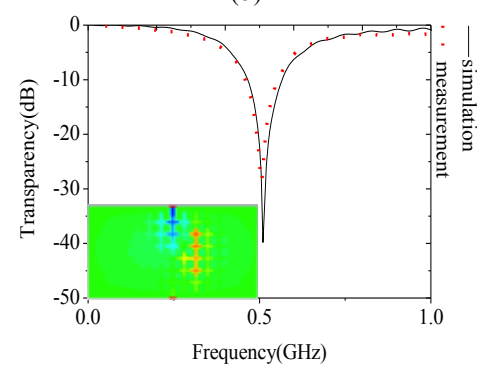

Figure 2: The plasma waveguide with defect length $\mathrm{L}_{2}=30 \mathrm{~mm}$ at the right side (a) and its transmission spectrum (b). The inset shows its electric field distribution.

If the two defects with slightly different resonant frequencies exist simultaneously on the opposite side of the DPS materials (shown in figure 3 (a)), the EIT-like behavior would show up in transmission spectra. As shown in figure 3 (b), the transparency is about equal to $-5 \mathrm{~dB}$ at $0.55 \mathrm{GHz}$. The transparency of $-5 \mathrm{~dB}$ reveals that the palsma waveguide supports the propagation of EM waves. And the result of CST simulation reveals that EM waves can pass the defective plasma waveguide because the two defects resonate with reversed phase at the frequency $0.55 \mathrm{GHz}$. It is the resonance of reversed phase that caused the reflected EM waves to cancel interference and caused the plasma waveguide to become transparent again.

(a)

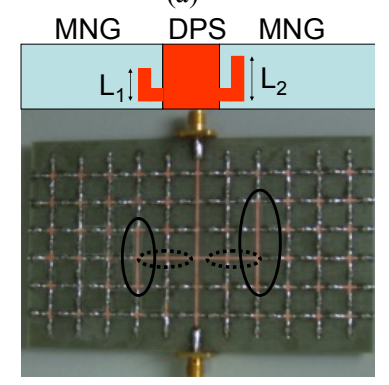

(b)

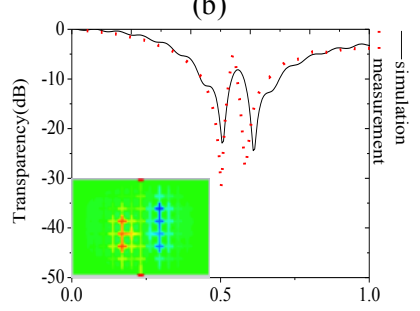

Figure 3: The EIT-like plasma waveguide (a) and its transmission spectrum (b). The inset shows its electric field distribution. 
To carry out a thorough study on the physics of the EITlike transmission in the above paper, the defects lengths were kept to be $20 \mathrm{~mm}$ and $30 \mathrm{~mm}$ respectively, but the left defect is moved $10 \mathrm{~mm}$ upward (figure 4 (a)). The results of simulation and measurement for the shift show that the EITlike transmission become worse (figure 4 (b), the EIT-like tansparency is about $-20 \mathrm{~dB}$ in measurement) and EM waves could not penetrate at the frequency of $0.49 \mathrm{GHz}$ (between $0.46 \mathrm{GHz}$ and $0.52 \mathrm{GHz}$ ), because the condition of the cancellation of opposite contributions from the two defects locating at the two sides of DSP is not satisfied well.

(a)

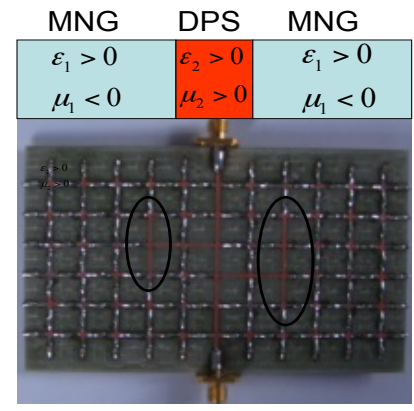

(b)

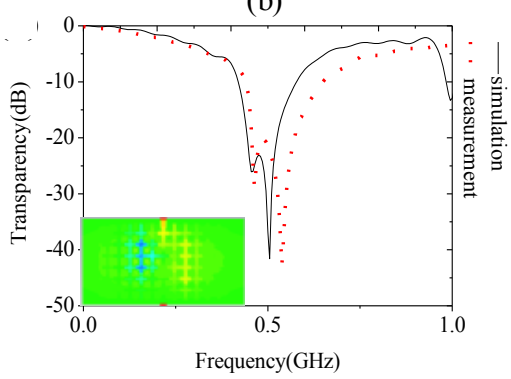

Figure 4: The plasma waveguide with the two defects moving $10 \mathrm{~mm}$ (a) at the both sides of DPS and its transmission spectrum. (b). The inset shows its electric field distribution.

\section{Conclusions}

In summary, the EIT-like phenomenon in the sandwich structure composed of defective MNG-DPS-MNG plasma waveguide has been observed in microwave regime. It is shown that the transmission peak can occur between the two resonators frequencies because of the destructive interference effect. And the EIT-like transmission becomes worse while one defect was moved. The measurement and simulation agree well with each other.

\section{Acknowledgements}

This research was supported by National Basic Research program (973) of China (No. 2011CB922001), by the NFSC (No.10904032) and by the Excellent Youth Teachers Program of Universities in Henan Province (2012GGJS060).

\section{References}

[1] F. Michael, Electromagnetically induced transparency: Optics in coherent media, Rev. Mod. Phys., Vol 77, No.2, pp.633-673, 2005.

[2] S. E. Harris, Electromagnetically Induc Transparency, Phys. Today 50, No. 7, pp.36-42, 1997.

[3] S. E. Harris, J. E. Field, and A. Imamoglu, Nonlinear optical processes using electromagnetically induced transparency, Phys. Rev. Lett., Vol 64, pp.1107-1110, 1990.

[4] F. Phillips, A. Fleischhauer, A. Mair, R. L. Walsworth, M. D. Lukin, Storage of Light in Atomic Vapor, Phys. Rev. Lett., Vol 86, 2001

[5] N Liu, T.Weiss, M. Mesch, L. Langguth,U. Eigenthaler, M. Hirscher, C. Sönnichsen, and H. Giessen, Planar metamaterial analogue of electromagnetically induced transparency for plasmonic sensing, Nano Lett., Vol 10, pp.1103-1107, 2010.

[6] S. Zhang, D. A. Genov, Y. Wang, M. Liu, and X. Zhang, Plasmon-induced transparency in metamaterials, Phys. Rev. Lett., Vol 101, 2008.

[7] C. Caloz, T. Itoh, Electromagnetic Metamaterials: Transmission Line Theory and Microwave Applications (Wiley \& Sons, New York, 2006.

[8] A. K. Iyer, P. C. Kremer and G. V. Eleftheriades, Experimental and theoretical verification of focusing in a large, periodically loaded transmission line negative refractive index metamaterial, Opt. Express ,Vol. 11, pp.696-707, 2003.

[9] R. Ruppin, Surface polaritons of a left-handed material slab, J. Phys.: Condens. Matter, Vol 13, pp.1811-1819, 2001. 